\title{
A Comparison of Transcutaneous and Total Serum Bilirubin in Newborn Hispanic Infants at 35 or More Weeks of Gestation
}

\author{
Karyn B. Kolman, MD, Katbleen M. Mathieson, PhD, and Carlos Frias, MD
}

Introduction: Transcutaneous bilirubin (TcB) measurement in newborns has been studied extensively in the non-Hispanic population, but its usefulness in the Hispanic population remains unclear. We evaluate the accuracy of TcB measurements in assessing jaundice in Hispanic neonates by using total serum bilirubin (TSB) measurements as the reference standard and determine the TcB level that can be used to identify neonates at risk for clinically significant jaundice (above the 95th percentile).

Methods: A total of 192 Hispanic neonates were enrolled. TcB measurements were performed within 30 minutes of obtaining the TSB measurement. The linear relationship between TcB and TSB was measured by using the Pearson correlation coefficient $(r)$. We calculated sensitivity, specificity, and positive and negative predictive values by using a TcB level above the 75th percentile to identify neonates with a TSB level above the 95th percentile.

Results: TSB ranged from 1.7 to $13.9 \mathrm{mg} / \mathrm{dL}$, with $62 \%$ falling below the 75 th percentile. TcB correlated well with TSB in Hispanic neonates $(r=0.87)$. A TcB level above the 75 th percentile detected all infants with a TSB level above the 95 th percentile, sensitivity $100 \%$, and specificity $66 \%$.

Conclusions: In Hispanic newborns, the TcB level correlated well with the TSB level. TcB monitoring is a useful clinical screening tool to evaluate for the risk of clinically significant jaundice. ( $\mathrm{J}$ Am Board Fam Med 2007;20:266-271.)

Neonatal jaundice, or hyperbilirubinemia, is a common problem encountered in the newborn nursery, occurring in approximately $65 \%$ of all fullterm babies with the peak bilirubin levels occurring on day 5 of life. ${ }^{1}$ Complications of hyperbilirubinemia, such as acute bilirubin encephalopathy and/or kernicterus, are rare in infants whose bilirubin levels are below the 95 th percentile. However, the preventability of these complications has led to recommendations to screen all neonates for hyperbilirubinemia. ${ }^{1-4}$ In July 2004, the American Academy of Pediatrics issued a clinical practice guideline, "Management of Hyperbilirubinemia in the Newborn Infant 35 or More Weeks of Gesta-

This article was externally peer reviewed.

Submitted 30 September 2006; revised 8 January 2007; accepted 11 January 2007.

From the Departments of Family and Community Medicine (KBK, CF) and Research (KMM), Maricopa Medical Center, Phoenix, AZ.

Funding: none.

Conflict of interest: none declared.

Corresponding author: Dr. Karyn B. Kolman, Department of Family and Community Medicine, Maricopa Medical Center, 601 East Roosevelt, Phoenix, AZ 85008 (E-mail: karyn_kolman@medprodoctors.com). tion." This guideline recommended that all newborn nurseries have a protocol for assessing jaundice. However, it did not specify the method by which jaundice must be assessed. Both TSB and $\mathrm{TcB}$ are cited as acceptable ways to assess neonatal jaundice. $^{4}$

Levels of TcB can be determined with a device that noninvasively estimates TSB levels by measuring light transmission through the skin of neonates. There are several TcB meters available, and previous studies of the various meters have shown that a linear relationship exists between $\mathrm{TcB}$ and TSB $[\mathrm{r}=0.87$ to 0.96$] .{ }^{5-10}$ However, at high levels of TSB $(>15 \mathrm{mg} / \mathrm{dL})$, the accuracy of TcB is less clear, but seems to be good. ${ }^{6,10}$ The literature does support using $\mathrm{TcB}$ as a tool for the initial assessment of neonatal jaundice as a means of decreasing the number of blood draws performed on neonates. If the $\mathrm{TcB}$ is high, a subsequent blood draw is then required for confirmation. ${ }^{5-14}$

The accuracy of $\mathrm{TcB}$ meters has been assessed in neonates from a variety of ethnic backgrounds including, African, Asian, and Indian. ${ }^{15-18}$ However, the data on Hispanic neonates are limited. Although Hispanic neonates were included in most 
studies, they comprised only a small percentage of the total study populations. ${ }^{5-7,10}$

Only 2 studies in the literature include a large percentage of neonates of Hispanic origin. The first study published in 2000, by Engle et al, ${ }^{19}$ found a poor correlation between $\mathrm{TcB}$ measurement and TSB levels in Hispanic neonates in the newborn nursery setting. However, this study focused on neonates with very high TSB levels (31\% had TSB $\geq 15 \mathrm{mg} / \mathrm{dL}$ ). A second study by the same group was published in $2005,{ }^{20}$ this time focusing on Hispanic neonates in the outpatient setting. This study found a better correlation of $\mathrm{TcB}$ and TSB, but again included a large percentage of the neonates with very high TSB levels (47\% had TSB $\geq 15 \mathrm{mg} / \mathrm{dL}$ ). Thus, the usefulness of TcB measurements in general population of newborn Hispanic infants remains unclear.

This study had 2 primary objectives: 1) To evaluate the accuracy of $\mathrm{TcB}$ measurements for assessing jaundice in the general population of Hispanic neonates, by using TSB as the reference standard; and 2) to determine the TcB level that can be used to identify neonates who are "at risk" for clinically significant jaundice, with risk defined as a TSB level above the 95th percentile.

\section{Methods}

\section{Setting}

The study was conducted in the newborn nursery at Maricopa Medical Center, a publicly funded district hospital in Phoenix, with approximately 3500 deliveries a year. The Maricopa Medical Center newborn nursery admits all healthy infants born at the hospital who are more than 35 weeks gestation and weigh more than $2267 \mathrm{~g}$. During the period of enrollment for this study (January through April 2006), approximately 800 infants were admitted to the Maricopa Medical Center nursery. More than $90 \%$ of infants admitted to the Maricopa Medical Center nursery are of Hispanic ethnicity, the majority of which are of Mexican descent.

The study was approved by the institutional review board at Maricopa Medical Center. Parents of all children received a written explanation of the study in both English and Spanish. Because this study was of a noninvasive intervention, the institutional review board required that verbal consent be obtained. The parents of all children in the study gave verbal informed consent.

\section{Subject Eligibility}

An infant was eligible for enrollment in the study if: 1) the infant was of Hispanic ethnicity, 2) the infant had not previously had a TSB level measured as part of this study, and 3) a trained nursery nurse was available to check a $\mathrm{TcB}$ measurement within 30 minutes of drawing a TSB level.

\section{Data Collection}

\section{Demographics}

Demographic data were collected by review of the infant's medical record. Hispanic race/ethnicity was verified by the race designation on the admission face sheet (as stated by the patient's mother at the time of registration) and/or the mother's primary language being designated as Spanish on the neonatal admission record.

\section{TSB Measurement}

All infants admitted to the MMC newborn nursery routinely undergo measurement of TSB before discharge from the nursery. TSB is measured earlier than the day of discharge if jaundice is suspected by clinical examination.

For TSB measurements, blood was obtained by venous puncture. TSB assays were performed in the clinical laboratory at Maricopa Medical Center using the Ortho VITROS 950 or the Ortho VITROS 5.1 FS Chemistry System (Ortho-Clinical Diagnostics, Rochester, NY). These analyzers measure TSB using a modified diazo reaction. The machines were calibrated daily according to the manufacturers' recommendations. The assays used by these machines were previously studied in comparison with the gold standard bilirubin measurement of high-profile liquid chromatography and were found to correlate well. ${ }^{21}$ In addition, one study compared high-performance liquid chromatography, modified diazo reactions, and $\mathrm{TcB}$ measurements and found good correlation among all 3 methods. ${ }^{6}$

For TcB measurements, we used the BiliCheck device. All measurements were performed with a single BiliCheck device in accordance with the manufacturer's recommendations. The BiliCheck $\mathrm{TcB}$ meter has been studied extensively. ${ }^{6,7,9,12,15,16,19}$ BiliCheck measures TcB using the entire spectrum of visible light (380 to $760 \mathrm{~nm}$ ). White light is transmitted through the skin of the neonate and by analysis of the light reflected back from the neonate's skin, an internal microprocessor 
calculates the amount of bilirubin present in the skin. The microprocessor uses an algorithm to account for interfering factors such as hemoglobin, melanin, and dermal thickness. As a result, theoretically, the BiliCheck should be able to provide an unbiased measurement of $\mathrm{TcB}$ independent of race/ethnicity. ${ }^{22}$

As noted, newborn nursery nurses obtained $\mathrm{TcB}$ measurements within 30 minutes of the TSB. At the onset of this study, all nurses obtaining TcB measurements received one-on-one instruction regarding the manufacturer's recommended method for obtaining $\mathrm{TcB}$ measurements. The device was calibrated before each measurement according to the manufacturer's recommendations. A total of 3 $\mathrm{TcB}$ measurements were obtained from each infant's forehead and these were averaged to obtain a single TcB measurement. The intraclass correlation coefficient for the 3 consecutive TcB readings for each infant was $0.87(P=.000)$, indicating strong test-retest reliability. Therefore, the average of these 3 measurements was used in subsequent analyses. We obtained only one TSB level and one averaged $\mathrm{TcB}$ for each infant.

\section{Risk Level Determination}

We used the bilirubin nomogram developed by Bhutani et $\mathrm{al}^{3}$ to divide bilirubin levels into 2 groups, clinically significant jaundice, and nonclinically significant jaundice. The bilirubin nomogram plots age in hours vs. bilirubin. As the level of bilirubin normally varies with age, this nomogram helps determine whether the level of bilirubin at a particular hour of life puts an infant at risk for developing clinically significant hyperbilirubinemia. If a bilirubin value is above the 95 th percentile for age curve, the nomogram predicts that the infant is in the "high-risk zone" for developing clinically significant hyperbilirubinemia. Similarly, bilirubin levels between the 75th and 95th percentile curves predict the infant is in the "high-intermediate risk zone."

Both the TSB and the averaged TcB values obtained for each infant were plotted on this nomogram. Because the TSB and TcB values for each individual infant were obtained at essentially the same time, the same hour of life was used to plot both values. For clinical significance, we used TSB values above the 95 th percentile and $T c B$ values above the 75 th percentile. ${ }^{6,19}$ All bilirubin samples were obtained during the ages of life that are included in the nomogram (0 to 144 hours old).

\section{Data Analysis}

Previous research demonstrated a high correlation $(r \sim 0.90)$ between total serum bilirubin and transcutaneous bilirubin measurements in non-Hispanic infants. To account for the possibility that the correlation may be slightly less in Hispanic neonates, we based our sample size calculation on the assumption that there would be a correlation of 0.85 between serum and transcutaneous levels in Hispanic infants. Assuming this 0.85 correlation, and assuming 95\% confidence (2-tailed) and 80\% power, 190 patients were needed for this study.

Data were entered into a Microsoft Excel spreadsheet and analyzed using SPSS version 14.0.

The overall relationship between TcB and TSB measures was assessed using the Pearson product moment correlation $(r)$, regression slope $(\beta)$, and Bland and Altman error plots. ${ }^{23}$ Sensitivity, specificity, and positive and negative predictive values were calculated.

\section{Results \\ Subjects}

A total of 198 admitted infants were enrolled. Six of these infants were eventually excluded from analysis when it was found that their race/ethnicity was not Hispanic. In total, therefore, 192 infants were included in our study.

Mean gestational age was 39 weeks and mean birth weight was $3369 \mathrm{~g}$. Mean age at the time when measurements were taken was 40 hours.

\section{Correlations}

TSB values ranged from 1.7 to $13.9 \mathrm{mg} / \mathrm{dL}$, with $62 \%$ of neonates below the 75 th percentile for age, $31 \%$ with levels between the 75 th and 95 th percentiles for age, and $6 \%$ with levels above the 95 th percentile for age (Table 1).

TcB correlated well with TSB $(r=0.87,95 \%$ confidence interval $[\mathrm{CI}]=0.84$ to 0.89$)$. Fig. 1 shows the regression plot of TSB and TCB. The slope $(\beta)$ of the regression plot is 0.97 , illustrating the strong correlation between $\mathrm{TcB}$ and TSB. Overall, TcB slightly underestimated TSB, as suggested by the small offset, or $Y$ intercept of the regression plot $(y=0.27)$. 
Table 1. Sample Characteristics

\begin{tabular}{lc}
\hline Characteristic & Mean (SD) or n (\%) \\
\hline Gestational age (weeks) & $39(1.5)$ \\
Birth weight (grams) & $3368(489.4)$ \\
Age at time of measurements (hours) & $40(13.4)$ \\
Serum bilirubin for age & \\
$\quad$ below 75th percentile & $120(62.5 \%)$ \\
75th to 95th percentile & $60(31.3 \%)$ \\
above 95th percentile & $12(6.3 \%)$ \\
\hline
\end{tabular}

The average error in evaluating hyperbilirubinemia with $\mathrm{TcB}$, compared with evaluation with TSB (calculated by TSB - TcB), was $0.06 \mathrm{mg} / \mathrm{dL}$ (95\% CI $=-0.12$ to 0.23$)$. A Bland-Altman error plot shown in Fig. 2, illustrates that only 9 data points (5\%) fall outside 2 standard deviations of the difference, indicating that the differences are normally distributed.

\section{Sensitivity and Specificity}

None of the 119 infants with TcB below the 75th percentile for age had clinically significant hyperbilirubinemia (TSB above the 95th percentile for age). The sensitivity of $\mathrm{TcB}$ measurements for detecting this level of hyperbilirubinemia was thus $100 \%$. A total of 73 infants had TcB above the 75 th percentile for age. Of these, only 12 had clinically significant jaundice with TSB level above the 95th percentile for age. Thus, the specificity was $66 \%$ for detecting clinically significant hyperbilirubinemia (Table 2).

\section{Discussion}

The BiliCheck TcB meter has been studied extensively, $6,7,9,12,15,16,19$ but its usefulness in the Hispanic population became questionable with the publication of a study done in 2000 at Parkland Hospital by Engle et al. ${ }^{19}$ This study found that there was a poor correlation between $\mathrm{TcB}$ measurements and TSB levels in Hispanic neonates. However, because the Engle study included a relatively large percentage of infants with very high TSB levels $(\geq 15)$, the usefulness of TcB measurements in the general population of Hispanic, most of whom would have a normal or mildly elevated bilirubin level, remained unclear.

Our study investigated the correlation of $\mathrm{TcB}$ levels with TSB levels in the Hispanic population with the intent of using $\mathrm{TcB}$ levels as a screening tool for clinically significant (above the 95th percentile) neonatal hyperbilirubinemia. According to our results, in the Hispanic population, as in other ethnic groups, TcB levels correlate well with the gold standard measurement of TSB. Furthermore, our study demonstrated that using a TcB level of above the 75 th percentile for age was $100 \%$ sensitive in iden-

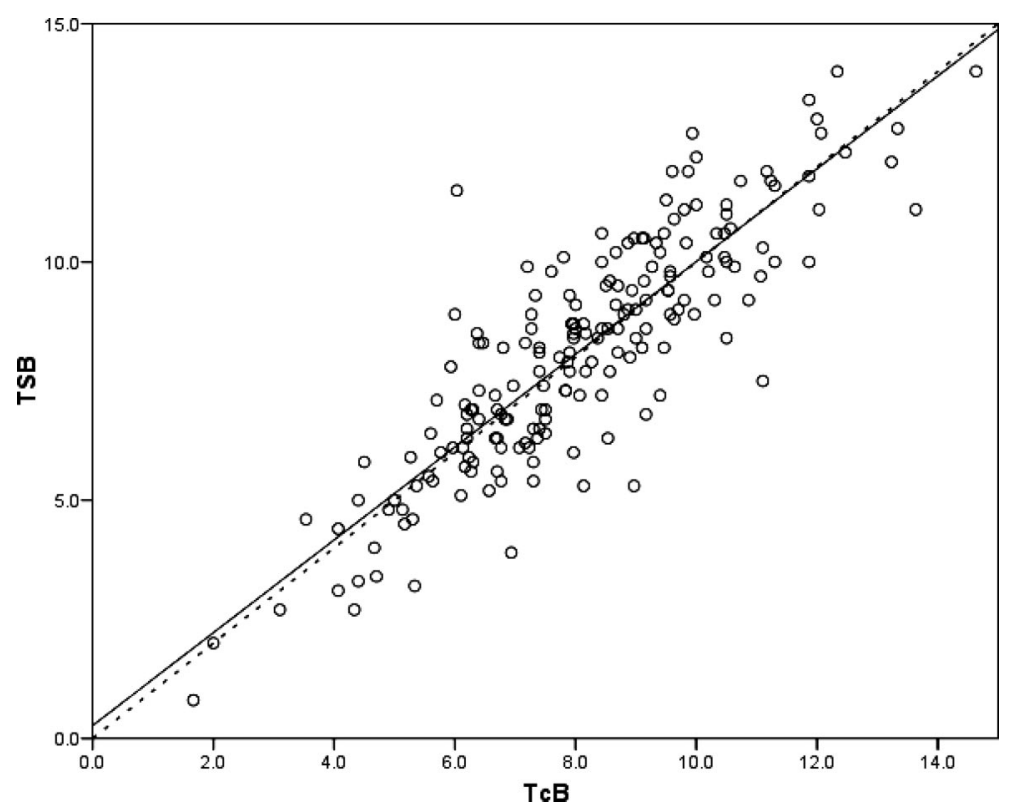

Figure 1. Total serum bilirubin versus transcutaneous bilirubin measurements. $N=192$ comparisons. The line of equality $(\mathrm{TcB}=\mathrm{TSB})$ is represented by the dotted line. The equation for the least squares best fit line is TSB $=$ $.271+.974(\mathrm{TcB})$, represented by the solid line. 


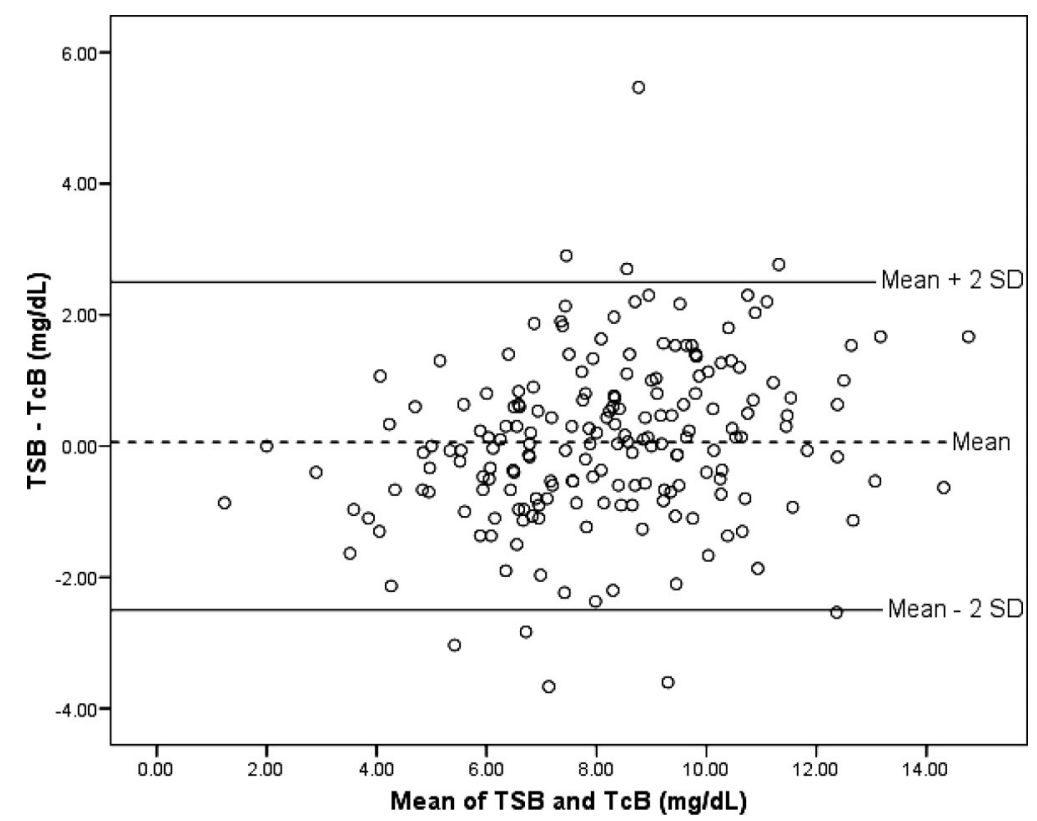

Figure 2. Error distribution plot of total serum bilirubin and transcutaneous bilirubin. Mean difference between TSB and TcB, plotted against mean of TSB and TcB.

tifying infants who may be at risk for clinically significant hyperbilirubinemia.

As early discharge from the hospital is becoming more frequent, there has been increasing concern that infants may develop serious sequelae of unrecognized hyperbilirubinemia. This concern has led to a number of recommendations for universal screening for neonatal hyperbilirubinemia, and ultimately resulted in the publication of the 2004 American Academy of Pediatrics guideline for management of neonatal hyperbilirubinemia., ${ }^{2,3}$ The guideline recommended a predischarge riskassessment for the development of severe hyperbilirubinemia in every newborn. At the time the guideline was published, transcutaneous bilirubinometry had already been developed, but its use was not universal. With the call for universal

Table 2. Predictive Indices Using $>$ 95th Percentile TSB and $\geq 75$ th Percentile TcB*

\begin{tabular}{lcc}
\hline & $\begin{array}{c}\text { TSB } \\
\text { >95 th Percentile }\end{array}$ & $\begin{array}{c}\text { TSB } \\
\leq 95 \text { th Percentile }\end{array}$ \\
\hline TcB & & \\
$\geq 75$ th percentile & 12 & 61 \\
$<75$ th percentile & 0 & 119 \\
Total $(\mathrm{N}=192)$ & 12 & 180 \\
\hline
\end{tabular}

${ }^{*}$ For all values, sensitivity $=100 \%$, specificity $=66.1 \%$, positive predictive value $=16.4 \%$, and negative predictive value $=100 \%$. screening the value of an effective, safe, and noninvasive tool for the evaluation of neonatal hyperbilirubinemia grew.

The results of our study support the use of the $\mathrm{TcB}$ measurement as a first-line tool for hyperbilirubinemia screening. If an infant has a $\mathrm{TcB}$ level above the 75 th percentile for age, it is safe to assume that the infant has a low risk of developing clinically significant hyperbilirubinemia, and no further testing need be performed. In our nursery, where every newborn is required to have a TSB level checked before discharge, this would reduce the number of TSB measurements, and hence a potentially painful blood draw, by $60 \%$. In addition, the use of $\mathrm{TcB}$ as a first-line screening tool also might result in a significant health-care cost reduction. At the time of the study, the average cost (charged to the patient) of a TcB and TSB was $\$ 4$ and $\$ 15$, respectively (a $73 \%$ cost difference).

\section{Limitations}

Our study has several limitations that should be considered when interpreting the results.

First, because of nursing staff considerations, the infants in our study were not enrolled consecutively or randomly. Instead, infants were only enrolled in the study when appropriately trained nursing staff was available. Because the infants were not sampled consecutively, the potential for selection bias is 
possible. The magnitude and direction of this bias, if it occurred, cannot be determined.

Next, because our study focused on normal newborns, the results of this study may not be applicable to Hispanic neonates who are preterm, have low birth weight, or have other risk factors for hyperbilirubinemia such as infection.

In summary, the results of this study support the use of the transcutaneous bilirubin meter as a screening tool for clinically significant hyperbilirubinemia in Hispanic infants. The TcB meter had excellent sensitivity, making it unlikely that an infant with a significant level of hyperbilirubinemia would escape detection with $\mathrm{TcB}$ measurement. Although the specificity of the TcB level was only $66 \%$, the test was able to identify a sizeable group of infants that did not need invasive testing for the evaluation of hyperbilirubinemia. Based on our results, we recommend that all normal, term, Hispanic infants be screened for hyperbilirubinemia initially with the measurement of $\mathrm{TcB}$ level. If the results indicate a TcB level above the 75 th percentile for age, a TSB level should be obtained.

We acknowledge the support of the nursing staff of the newborn nursery at Maricopa Medical Center and their assistance with data collection.

\section{References}

1. Maisels MD. Neonatal Jaundice. In: Effective Care of the Newborn. Sinclair JC, Bracken MB, eds. Oxford: Oxford University Press; 1993. p. 507-62.

2. Johnson LH, Bhutani VK. System-based approach to management of neonatal jaundice and prevention of kernicterus. Pediatrics 2002;140:393-403.

3. American Academy of Pediatrics, Subcommittee on Hyperbilirubinemia. Management of hyperbilirubinemia in the newborn infant 35 or more weeks of gestation. Pediatrics 2004;114:297-316.

4. Ip S, Chung M, Kulig J, et al. An evidence-based review of important issues concerning neonatal hyperbilirubinemia. Pediatrics 2004;114:130-53.

5. Tayaba R, Gribetz D, Gribetz I, Holzman IR. Noninvasive estimation of serum bilirubin. Pediatrics 1998;102:e28.

6. Bhutani VK, Gourley GR, Adler S, Kreamer B, Dalin C, Johnson LH. Nonivasive measurement of total serum bilirubin in a multiracial predischarge newborn population to assess the risk of severe hyperbilirubinemia. Pediatrics 2000;107:e17.

7. Rubaltelli FF, Gourley GR, Luskamp N, et al. Transcutaneous bilirubin measurement: a multicenter evaluation of a new device. Pediatrics 2001; 107:1264-71.
8. Carbonell X, Butet F, Figueras J, Rui-Godo A. Prediction of hyperbilirubinemia in the healthy term newborn. Acta Paediatr 2001;90:166-70.

9. Ebbesen F, Rasmussen LM, Wimberley PD. A new transcutaneous bilirubinometer, BiliCheck, used in the neonatal intensive care unit and the maternity ward. Acta Paediatr 2002;91:203-11.

10. Maisels MJ, Ostrea EM, Touch S, et al. Evaluation of a new transcutaneous bilirubinometer. Pediatrics 2004; 113:1628-35.

11. Briscoe L, Clark S, Yoxal CW. Can transcutaneous bilirubinometry reduce the need for blood tests in jaundiced full term babies? Arch Dis Child Fetal Neonatal Ed 2002;86:F190-2.

12. Nanjundaswamy S, Petrova A, Mehta R, Bernstein $W$, Hegyi T. The accuracy of transcutaneous bilirubin measurements in neonates: a correlation study. Biol Neonat 2004;85:21-5.

13. Yap SH, Mohammad I, Ryan CA. Avoiding painful blood sampling in neonates by transcutaneous bilirubinometry. Ir J Med Sci 2002;171:188-90.

14. Maisels MJ, Kring E. Transcutaneous bilirubinometry decreases the need for serum bilirubin measurements and saves money. Pediatrics 1997;99:599-601.

15. Slusher TM, Angyo IA, Bode-Thomas F, et al. Transcutaneous bilirubin measurements and serum total bilirubin levels in indigenous African infants. Pediatrics 2004;113:1636-41.

16. Janjindamai $W$, Tansantiwong T. Accuracy of transcutaneous bilirubinometer estimates using BiliCheck in Thai neonates. J Med Assoc Thai 2005;88:187-90.

17. Yamauchi Y, Yamanouchi I. Transcutaneous bilirubinometry in normal Japanese infants. Acta Paediatr Jpn 1989;31:65-72.

18. Bhat V, Srinivasan S, Usha TS, Puri RK. Correlation of transcutaneous bilirubinometry with serum bilirubin in South Indian neonates. Indian J Med Res 1987;86:49-52.

19. Engle WD, Jackson GL, Sendelbach D, Manning D, Frawley WH. Assessment of a transcutaneous device in the evaluation of neonatal hyperbilirubinemia in a primarily Hispanic population. Pediatrics 2002;110:61-7.

20. Engle ED, Jackson GL, Stehel EK, Sendelbach D, Manning MD. Evaluation of a transcutaneous jaundice meter following hospital discharge in term and near-term neonates. J Perinatol 2005;25:486-90.

21. Gourley GR, Bhutani V, Johnson L, Kreamer B, Kosorok MR, Dalin C. Measurement of serum bilirubin in newborn infants: common clinical laboratory methods versus high performance liquid chromatography (HPLC). Pediatr Res 1999;45:283A.

22. Bertini G, Rubaltelli FF. Non-invansive bilirubinometry in neonatal jaundice. Semin Neonatol 2002; 7:129-33.

23. Bland JM, Altman DG. Statistical methods for assessing agreement between two methods of clinical measurement. Lancet 1986;1:307-10. 